\title{
Management of traumatic brain injury in the intensive care unit: A useful mnemonic and a review of the literature.
}

\section{Tobi KU*}

Department of Surgery and Anaesthesiology, School of Medicine, Hage Geingob Campus, University of Namibia, Windhoek, Republic of Namibia

\begin{abstract}
Traumatic brain injury (TBI) accounts for about 30-50\% of all trauma-related deaths with a male predominance. It can be classified on the basis of neurological assessment using Glasgow Coma Score into, mild (13/15), moderate (9-12/15) and severe (3-8/15). Moderate to severe TBI often require admission to the Intensive care unit (ICU). Mnemonics have been found to be useful in remembering systematic management of clinical conditions especially for the young and inexperienced physician. This is an attempt to provide a useful mnemonic, "HEAD" for the management of TBI in the ICU.
\end{abstract}

Keywords: Traumatic brain injury, Intensive care unit, Hyperthermia, Mortality.

\section{Introduction}

Traumatic brain injury (TBI) accounts for about 30-50\% of all trauma-related deaths with a male predominance [1]. It can be classified on the basis of neurological assessment using Glasgow Coma Score into, mild (13/15), moderate (9-12/15) and severe (3-8/15). Moderate to severe TBI often require admission to the Intensive care unit (ICU).

The aetiology of TBI includes but not limited to road traffic injury, falls from height, industrial accidents and assaults. It has been estimated that about $30 \%$ of deaths from TBI occur within the first six hours of injury and more than $50 \%$ with the first 24 hours [2]. Immediate admission and management of TBI patients in the intensive care unit reduces morbidity and mortality.

The pathophysiology of TBI involves intracranial inflammatory response with release of mediators such as cytokines and free radicals which alter brain blood barrier permeability. There is also a disruption of cerebral autoregulation with hyperperfusion phase in the first 72 hours, hyperemic phase between 7-10 days and vasospastic phase which develop later on [2].

Management of moderate to severe TBI usually starts in the emergency room with initial resuscitation before transfer to the Intensive Care Unit (ICU). The goal of ICU management of TBI is the prevention of secondary brain injuries such as hypotension, hypercapnia, hypertension, hypo/hyperglycaemia and hyperthermia. In addition, morbidity and/or mortality following TBI is determined by the severity and presence of these secondary injuries [3].

This is an attempt to provide a useful mnemonic, "HEAD" for the management of TBI in the ICU.

\section{Literature Review}

\section{Resuscitation}

Initial assessment: at the emergency room
Airway: Patients with a $\mathrm{GCS}<8 / 15$ or at risk of further deterioration with subsequent loss of the airway require endotracheal intubation using a rapid sequence induction with cricoid pressure and in-line mobilization of the $\mathrm{C}$-spine and immobilize with a rigid cervical collar.

Breathing: Patients will often require oxygen supplementation or mechanical ventilation with $100 \%$ oxygen using a tidal volume of $5-7 \mathrm{ml} / \mathrm{kg}$ until Arterial blood gas (ABG) analysis is available. With the availability of blood gas values, appropriate ventilator setting with $\mathrm{FiO}_{2}$ will be imputed. The aim of ventilation in TBI is to avoid hypoxia which is a secondary injury.

Circulation: Assessment of volume status and restoration of circulating blood volume is required to ensure euvolemia and to maximize oxygenation. Direct arterial blood pressure and Central venous pressure (CVP) monitoring may be indicated. Inotropes/vasopressors may be needed to maintain Cerebral perfusion pressure (CPP) at $>70 \mathrm{mmHg}$ and systolic blood pressure $>100 \mathrm{mmHg}$.

Disability: Assessment of the level of consciousness (GCS/ AVPU), pupillary reflexes and motor functions is done and documented. Any change in the level of consciousness should be noted promptly and appropriate steps instituted such as emergency craniotomy.

\section{Imaging in TBI}

Patients with TBI are often taken to the radiological suite for different types of imaging depending on their clinical states.

X-rays: Routine trauma series of X-rays namely: chest, pelvis, and C-spine (AP, lateral, and peg views). This is to rule out or confirm other associated injuries that may require urgent medical or surgical interventions.

Computer tomography (CT) scanning: This is the most informative radiological test and is the standard for traumatic 
brain head injury in virtually all patients. The most important role of CT scanning is the prompt detection of mass lesions as an extradural or subdural haematoma.

Cerebral angiography: Should be considered when a vascular injury such as carotid artery dissection is suspected in head-injured patients.

\section{ICU management ("HEAD")}

The following outline vis-à-vis the acronym "HEAD" has been developed by the author to help practitioners and students manage patients with TBI in a systematic manner without leaving out any details. It has been observed that protocol management of severe brain injury in the ICU aids favourable outcome.

\section{H: Avoiding the "Hs" i.e.,}

Hypotension: (Maintain MAP $>90 \mathrm{mmHg}, \quad \mathrm{SBP}>100$ $\mathrm{mmHg}$ ). Hypotension which is defined as Systolic blood pressure (SBP) less than $90 \mathrm{mmHg}$ is significantly associated with increased mortality following TBI [4]. Aggressive fluid administration under Central venous pressure (CVP) monitoring $\left(8-10 \mathrm{~cm} \mathrm{H}_{2} \mathrm{O}\right)$ is the first step in the prevention and management of hypotension in these patients. After adequate volume resuscitation, patient who remain hypotensive may be given vasopressors or inotrope to maintain MAP $>65 \mathrm{mmHg}$.

Hypoxia: (Maintain $\mathrm{SpO}_{2}>95 \%$ and $\mathrm{PaO}_{2}>75 \mathrm{mmHg}$ ). Hypoxia is defined as oxygen saturation less than $90 \%$, or $\mathrm{PaO}_{2}<60$ $\mathrm{mmHg}$. Adequate oxygenation as defined above is ensured with the means of mechanical ventilation with appropriate setting and regular $\mathrm{ABG}$ measurements. While ensuring adequate $\mathrm{SpO}_{2}$, hyperventilation should be cautiously avoided as it has been observed to increase e volume of severely hypoperfused tissue within the injured brain, despite improvements in cerebral perfusion pressure (CPP) and Intracranial pressure (ICP) [5]. In head injury, protective ventilation with low tidal volume and moderate Positive end-expiratory pressure (PEEP) has been recommended [6].

Hyperthermia: (Maintain temperature $<37.5^{\circ} \mathrm{C}$ with paracetamol and/or active cooling). Temperature control to below $37.5^{\circ} \mathrm{C}$ is indicated in patients with TBI with aggressive treatment of hyperthermia/fever. Although it has been observed that moderate systemic hypothermia $\left(32^{\circ} \mathrm{C}\right.$ to $\left.34^{\circ} \mathrm{C}\right)$, reduces cerebral metabolism and Cerebral blood volume (CBV), decreases Intracranial pressure (ICP), and increases Cerebral perfusion pressure (CPP) [7], there is no documented evidence to suggest its superiority over normothermia in the management of severe TBI [8].

Hyperglycaemia: (Maintain blood sugar $<10 \mathrm{mmol} / \mathrm{l}$ ). In patients with severe TBI, hyperglycemia is associated with poor neurological outcome [9]. Indeed, both hyperglycaemia and hypoglycaemia are detrimental to the injured brain, thus tight glycaemic control with the propensity for episodic hypoglycaemia has fallen into disrepute [10].

E: Elevate head of the bed to 30-45 degree.

End of bed elevation to $30^{\circ}-45^{\circ}$ with the head and neck of the patient in a neutral position reduces Intracranial pressure (ICP) and improves Cerebral perfusion pressure (CPP) [11]. Furthermore, this has also been found to reduce the risk of Ventilator-associated pneumonia (VAP).

A: Avoid agitation by adequate sedation with midazolam/ fentanyl combination.

Adequate sedation for the head injured patient in the ICU has many advantages as it improves analgesics, reduces anxiety and agitation. In addition, it allows instrumentation and mechanical ventilation, decreases oxygen consumption, carbon dioxide production and improves patient comfort [11]. Avoid stopping sedation in the first 48 hours of ICU admission. Commonly used sedatives include: Fentanyl at $2 \mathrm{mcg} / \mathrm{kg}$ test dose, 2-5 $\mathrm{mcg} / \mathrm{kg} / \mathrm{h}$ continuous infusion, Midazolam @ 2 mg test dose, 2-4 mg/h continuous infusion, Sufentanil 10-30 mcg test bolus, $0.05-2 \mathrm{mcg} / \mathrm{kg}$ continuous infusion and propofol at $0.5 \mathrm{mg} / \mathrm{kg}$ continuous infusion (not to exceed $5 \mathrm{mg} / \mathrm{kg} / \mathrm{h}$ ) [12].

\section{D: Drugs}

These include prophylactic broad spectrum antibiotics, antiseizures like phenytoin $(5 \mathrm{mg} / \mathrm{kg} /$ day $)$ or valproate $(15 \mathrm{mg} / \mathrm{kg} /$ day) for at least a week in all patients. It is to be noted that prophylactic use of phenytoin or valproate is not recommended for preventing late posttraumatic seizures. However, their use in the first week following injury decreases the incidence of early posttraumatic seizures [12]. Others are early feeding with good caloric intake (30-50 kcal/kg/day) and protein intake of $2 \mathrm{~g} / \mathrm{kg} /$ day should be maintained. Nutritional support should commence as early 48-72 hours after injury, blood transfusion if haemoglobin concentration $<10 \mathrm{~g} / \mathrm{dl}$ in the first 48 hours' postinjury, opioids in combination with NSAIDS.

\section{D: Deep venous thrombosis (DVT) prophylaxis}

TBI patients are prone to DVT and as such require prophylaxis which can be pharmacological or non-pharmacological. Nonpharmacological DVT prophylaxis can be achieved with the use of intermittent pneumatic compression stockings or graduated compression stock except in lower limb injuries and continued till the patient is ambulatory. Pharmacological method is with the administration of low-molecular-weight heparin or low-dose unfractionated heparin which could be used in combination with mechanical prophylaxis when it is safe, preferably after $72 \mathrm{~h}$ of intracranial haemorrhage/craniotomy with close monitoring and repeat brain $\mathrm{CT}$ to detect expansion of haematoma [12].

\section{Management of raised intracranial pressure}

Management of raised intracranial pressure in TBI patients begins with ICP monitoring when indicated and if the facility for it is available. It has been recommended that "Intracranial pressure (ICP) should be monitored in all salvageable patients with a severe TBI and an abnormal Computed tomography (CT) scan". Also, "ICP monitoring is indicated in patients with severe TBI with a normal CT scan if two or more of the following features are noted at admission: age over 40 years, unilateral or bilateral motor posturing, or systolic Blood pressure $(\mathrm{BP})<90$ mmHg" [13]. 


\section{Indications for ICP monitoring include ("HEAD")}

- Haemodynamic instability $\mathrm{SBP}<90 \mathrm{mmHG}$

- Severe head injury, GCS 3-8

- Age $>40$ years

- Decorticate/Decerebrate posturing

The gold standard for monitoring of ICP is the use of ventricular ICP catheter. It is the most accurate, cheap, and reliable method. Its other advantages are continuous measurement of ICP and for therapeutic drainage of Cerebrospinal fluid (CSF) to control raised ICP. In addition, drained CSF could be sent to the laboratory for analysis vis-à-vis microscopy/culture/sensitivity, glucose, protein, cell count and gram staining. One common disadvantage of ventricular catheter use for ICP monitoring is the risk of infection although prophylactic antibiotic use for is not a common practice [13].

\section{Treatment of raised ICP is ("HEAD")}

The critical ICP value for treatment is $>20 \mathrm{mmHg}$. In addition to this, the clinical and brain CT findings are essential consideration in determining the need for treatment [13].

Treatment of raised ICP can be summarized with the above acronym "HEAD" as follows:

H: Hypothermia to lower CBV and CBF thus ICP and Hyperventilation to a $\mathrm{PaCO}_{2}$ of $30-35 \mathrm{mmHg}$. $\mathrm{PaCO}_{2}<30$ $\mathrm{mmHg}$ should be avoided as stated earlier

E: Elevation of the head to avoid venous congestion and promote venous drainage from the head and subsequent reduction in ICP.

A: Adequate analgesia and sedation prevents raised ICP due to pain and discomfort.

D: Decompressive craniectomy. If the patient continues to have persistently raised ICP $(>20 \mathrm{mmHg})$ in spite of maximal medical management, decompressive craniectomy is the surgical available option to decrease ICP.

D: Diuretics (20\% mannitol and hypertonic saline).

Mannitol is effective for the control of raised ICP at doses of $0.25-1 \mathrm{~g} / \mathrm{kg}$ body weight over 15 minutes and it should be given only after CT scan to rule out extradural haematoma. Some side effects of mannitol include intravascular dehydration, hypotension, pre-renal azotemia and hyperkalemia [14]. It may also cause a reverse osmotic shift or rebound effect, and raising brain osmolarity, thus increasing ICP [15].

D: Drugs e.g. Barbiturate is effective for the management of refractory intracranial hypertension as it reduces cerebral metabolism and CBF, and thus lower ICP [16]. Induction of barbiturate come is only indicated in haemodynamically stable patients with refractory elevated ICP despite medical and surgical intervention. Side effects include hypotension and immunosuppression with an increased infection rate [17]. Regimen for inducing barbiturate coma: Pentobarbital: $10 \mathrm{mg} /$ $\mathrm{kg}$ over $30 \mathrm{~min}$, then $5 \mathrm{mg} / \mathrm{kg} / \mathrm{h}$ for 3 hours, then $1 \mathrm{mg} / \mathrm{kg} / \mathrm{h}$ or sodium thiopentone @ 2.5-10 mg/kg IV, slow bolus, then 0.5-2 $\mathrm{mg} / \mathrm{kg} / \mathrm{h}$ [13].

\section{Monitoring}

Regular monitoring of patients with severe TBI in the ICU is critical for the early detection and diagnosis and management of secondary brain injuries. These include:

Clinical assessment: Which involves regular assessment of the GCS, pupillary signs and motor responses which should recorded in the ICU chart.

ICP monitoring: Measurement of ICP with an intraventricular catheter is the most accurate and clinically useful method as mentioned earlier. Some of the advantages include zero calibration, CSF drainage for raised ICP, allows dynamic testing of pressure/vol relationship. It is however technically difficult with an increased incidence of infection.

Cerebral blood flow with jugular bulb oximetry: Measurement of $\mathrm{SjO} 2$ in the jugular bulb by the retrograde placement of a fibreoptic catheter provides an indirect assessment of cerebral perfusion. $\mathrm{SjO} 2<55 \%$ is suggestive of cerebral hypoperfusion, $\mathrm{SjO} 2>60 \%$ is suggestive of hyperemia [18].

Cerebral lactate fluxes: Other applications of jugular bulb catheter include calculation of cerebral lactate fluxes from intermittent blood samples. An arterio-jugular lactate difference of $-0.4 \mathrm{mmol} / \mathrm{l}$ or a lactate oxygen index AJLD/arteio-jugular saturation difference of $>0.08$ is suggestive of cerebral ischaemia or infarction [19].

Near-infrared spectroscopy: A non-invasive method of measuring regional cerebral oxygen saturations and cerebral blood volume using a scalp oximeter similar to pulse oximetry. Its principle is based the differential absorption properties of the two main chromophores in the brain namely Haemoglobin $\mathrm{Hb}$ ) and cytochrome oxidase in the near infrared range between 700 and $1,000 \mathrm{~nm}$ which can provide the degree of tissue deoxygenation [20].

Transcranial Doppler is a non-invasive method of measuring $\mathrm{CBF}$ velocity and it is useful in the diagnosis of vasospasm, critical elevations of ICP and decreases in CPP, carotid dissection, and brain death with an overall sensitivity and specificity of $75 \%$ to $88 \%$ and $98 \%$ respectively [21]. It can also predict posttraumatic vasospasm prior to its clinical manifestations and it has been suggested as a non-invasive alternative technique for assessment of ICP and CPP [22].

\section{Outcome in TBI}

Outcome and prognosis of TBI: Patient factors that determine the outcome include:

- The severity of primary and secondary injuries.

- Low Glasgow coma scale (GCS) on presentation.

- Advanced age $(>65)$

- Comorbidities. 


\section{The extended Glasgow outcome scale}

A blunt tool that is widely used to assess outcome from head injury. Takes the form of structured interviews with the patient or their caregivers and should be performed at 6 and 12 months post-injury [23].

\section{Conclusion}

The management of TBI in the ICU could be very challenging to the young and inexperienced physician. An easily remembered mnemonics such as presented here will serve as guide for the initial care and stabilization of this group of patients.

\section{References}

1. Tobi KU, Azeez AL, Agbedia SO. Outcome of traumatic brain injury in the intensive care unit: a five-year review. S Afr J Anaesth Analg. 2016;22(5):135-9.

2. McGlinch BP. Anesthesia for trauma and emergency surgery. In: Butterworth JF, Mackey DC, Wasnick JD, editors. Morgan and Mikhail's Clinical Anesthesiology. McGraw Hill Education Lange; 2013.

3. Unterberg AW, Stover JF, Kress B et al. Edema and brain trauma. Neurosci. 2004;129(4):1021-9.

4. Manley G, Knudson MM, Morabito D, et al. Hypotension, hypoxia, and head injury: frequency, duration, and consequences. Arch Surg. 2001;136(10):1118-23.

5. Coles JP, Minhas PS, Fryer TD, et al. Effect of hyperventilation on cerebral blood flow in traumatic head injury: clinical relevance and monitoring correlates. Crit Care Med. 2002;30(9):1950-9.

6. Mascia L, Grasso S, Fiore T, et al. Cerebro-pulmonary interactions during the application of low levels of positive end-expiratory pressure. Intensive Care Med. 2005;31(3):373-9.

7. Shiozaki T, Sugimoto H, Taneda M, et al. Effect of mild hypothermia on uncontrollable intracranial hypertension after severe head injury. J Neurosurg. 1993;79(3):363-8.

8. Clifton GL, Miller ER, Choi SC, et al. Lack of effect of induction of hypothermia after acute brain injury. New Engl J Med. 2001;344(8):556-63.

9. Lam AM, Winn HR, Cullen BF, et al. Hyperglycemia and neurological outcome in patients with head injury. $\mathrm{J}$ Neurosurg. 1991;75(4):545-51.

10. Marion DW. Optimum serum glucose levels for patients with severe traumatic brain injury. F 1000 Med Rep. 2009;1:42.

11. Ng I, Lim J, Wong HB. Effects of head posture on cerebral hemodynamics: its influences on intracranial pressure, cerebral perfusion pressure, and cerebral oxygenation. Neurosurgery. 2004;54(3):593-7.

12. Agrawal D. Severe Head and Spinal Cord Injury. In: Chawla R, Todi S editors. ICU protocols: stepwise approach. New York: Springer Dordrecht Heidelberg; 2012. p. 521-6.
13. Brain Trauma Foundation. Guidelines for the Management of Severe Traumatic Brain Injury. J Neurotrauma. 2007;24(1):S1-106.

14. Manninen PH, Lam AM, Gelb AW, et al. The effect of high dose mannitol on serum and urine electrolytes and osmolality in neurosurgical patients. Can J Anaesth. 1987;34(5):442-6.

15. Marshall LF, Smith RW, Rauscher LA, et al. Mannitol dose requirements in brain-injured patients. J Neurosurg. 1978;48(2):169-72.

16. Kassell NF, Hitchon PW, Gerk MK, et al. Alterations in cerebral blood flow, oxygen metabolism, and electrical activity produced by high dose sodium thiopental. Neurosurg. 1980;7(6):598-603.

17. Stover JF, Stocker R. Barbiturate coma may promote reversible bone marrow suppression in patients with severe isolated traumatic brain injury. Eur J Clin Pharmacol. 1998;54(7):529-34.

18. Robertson CS, Cormio M. Cerebral metabolic management. New Horiz. 1995;3(3):410-22.

19. Stahl N, Mellergard P, Hallstrom A, et al. Intracerebral microdialysis and bedside biochemical analysis in patients with fatal traumatic brain lesions. Acta Anaesthesiol Scand. 2001;45(8):977-85.

20. Haddad SH, Arabi YM. Critical care management of severe traumatic brain injury in adults. Scand J Trauma Resusc Emerg Med. 2012;20:12.

21. de Freitas GR, Andre C. Sensitivity of transcranial Doppler for confirming brain death: A prospective study of 270 cases. Acta Neurol Scand. 2006;113(6):426-32.

22. Ragauskas A, Daubaris G, Dziugys A et al. Innovative non-invasive method for absolute intracranial pressure measurement without calibration. Acta Neurochir Suppl. 2005;95:357-61.

23. Wilson JTL, Pettigrew LEL, Teasdale GM. Structured interviews for the Glasgow Outcome Scale and the Extended Glasgow Outcome Scale: Guidelines for Their Use. J Neurotrauma. 1997;15(8):573-85.

\section{*Correspondence to:}

\section{Tobi KU}

Senior Lecturer, Department of Surgery and Anaesthesiology

School of Medicine, Hage Geingob Campus, University of Namibia

Windhoek, Republic of Namibia

Tel: + 264812539227

E-mail: ktobi@unam.na; tobikingsley265@gmail. com; tobikingsley@yahoo.com 